\section{Tendência da hanseníase em menores de 15 anos no Brasil, 2001-2016}

\author{
Leprosy trends in children under 15 years of age \\ in Brazil, 2001-2016
}

\section{Tendencia de la hanseniasis en menores de 15 años en Brasil, 2001-2016}

Priscila Barros Schneider 1

Bruna Hinnah Borges Martins de Freitas 1

doi: 10.1590/0102-311X00101817

\section{Resumo}

O objetivo foi analisar a tendência das taxas de detecção de hanseníase em menores de 15 anos no Brasil, no periodo de 2001 a 2016. Trata-se de um estudo de série temporal, utilizando o procedimento de Prais-Winsten para análise de regressão linear generalizada com nível de significância de 5\%. A média da taxa de detecção foi de 5,77 por 100 mil habitantes. Entretanto, observou-se uma tendência decrescente dessa taxa, com annual percent change (APC) de -5\% (IC95\%: -6,7; -3,3). Verificou-se tendência decrescente em todas as regiões do país. Entre as regiões, a Norte foi a que manteve a média hiperendêmica ( $\geq 10,00$ por 100 mil habitantes) da taxa de detecção. A série temporal em 19 Unidades da Federação (UF) foi decrescente. Todavia, entre elas, muitas mantiveram média hiperendêmica, como: Mato Grosso, Pará, Maranhão, Rondônia, Roraima, Pernambuco, Piaui e Acre. Entre as oito UF que apresentaram tendência estacionária, Tocantins manteve média hiperendêmica no período. Das 24 capitais brasileiras incluídas no estudo, 14 delas foram decrescentes e 10 estacionárias. Embora com tendência decrescente dos casos novos, algumas capitais mantiveram a média hiperendêmica como: Teresina, Recife, Cuiabá, Boa Vista, Rio Branco e Belém. Apesar de apresentarem tendência estacionária, as capitais Palmas e São Luís registraram média hiperendêmica. Conclui-se que, embora a análise da tendência foi decrescente no Brasil, houve presença de tendências estacionárias e hiperendemicidade em algumas UF e capitais brasileiras, o que demonstra a permanência de fontes de transmissibilidade e dificuldade na eliminação da hanseníase no país.

Hanseníase; Criança; Adolescente; Distribuição Temporal

\author{
Correspondência \\ B. H. B. M. Freitas \\ Faculdade de Enfermagem, Universidade Federal de Mato \\ Grosso. \\ Av. Fernando Corrêa da Costa 2367, Cuiabá, MT \\ 78060-900, Brasil. \\ bruna_hinnah@hotmail.com \\ 1 Faculdade de Enfermagem, Universidade Federal de Mato \\ Grosso, Cuiabá, Brasil.
}




\section{Introdução}

A hanseníase é uma doença infectocontagiosa crônica, cujo agente etiológico é o Mycobacterium leprae, que tem alta infectividade e baixa patogenicidade. Essas propriedades não ocorrem apenas em função de suas características intrínsecas, mas também dependem da relação do agente com o hospedeiro e do grau de endemicidade do meio. A doença é transmitida pelo contato direto de um doente bacilífero com uma pessoa susceptível, principalmente através das vias aéreas superiores, sobretudo no ambiente domiciliar. Essa atinge a pele e os nervos periféricos, podendo causar deformidades e incapacidades físicas devido ao alto poder imunogênico do bacilo 1.

Dados da Organização Mundial da Saúde (OMS) apontam uma queda no número de casos novos no mundo entre 2005 e 2015, sendo que, o registro de casos novos em 2015 foi de 210.758. A Índia ocupou o primeiro lugar com 127.326 casos novos e o Brasil o segundo, com 26.395 casos. A proporção de menores de 15 anos acometida pela doença em 2015 equivaleu a 8,9\% dos casos novos no mundo e 7,35\% dos casos no Brasil 2.

A detecção de hanseníase em menores de 15 anos indica uma transmissão ativa e recente da infecção na comunidade. Por isso, desde 2011, o Ministério da Saúde, em consonância com os objetivos da OMS, atua por meio do Plano Integrado de Ações Estratégicas para Eliminação da Hanseníase. Esse tem o compromisso político de enfrentamento dessa doença negligenciada em prol da sua eliminação como problema de saúde pública, por meio do diagnóstico precoce e tratamento oportuno 1.

Essa faixa etária é marcada por um período de transição, com crescimento acelerado e desenvolvimento biopsicossocial. Nesse período, o indivíduo estabelece as relações sociais mais amplas e ocorre a redefinição do autoconceito. Doenças crônicas como a hanseníase, conhecida por suas lesões dermatológicas, deformidades e incapacidades físicas, podem interferir na imagem corporal e autoestima do indivíduo, influenciando na construção de sua identidade e, consequentemente, nas suas relações sociais. Além de ser capaz de comprometer sua escolarização, com um baixo rendimento escolar e até o abandono dos estudos por motivos de tratamento, discriminação e preconceito 3 .

A taxa de detecção de hanseníase nessa população é um importante indicador de fontes ativas da doença na população, mede a sua presença e a força de transmissão recente da endemia. Esse indicador permite acompanhar o alcance da eliminação da doença e auxilia no processo de tomada de decisão, contribuindo para a melhora dos processos organizacionais de vigilância epidemiológica 4 .

Um estudo internacional realizado em Kerala, na Índia, identificou que, em 10 anos (2004-2014), houve uma diminuição insignificante dos casos novos de hanseníase em menores de 15 anos. Porém, mostrou um aumento dos casos de pacientes que necessitavam de tratamento multibacilar e um declínio dos que necessitavam de tratamento paucibacilar 5 .

No Brasil, um estudo realizado em Fortaleza (Ceará) verificou uma tendência crescente de casos em um intervalo de 13 anos (1995-2008) com uma média de 95 casos/ano 6. Contudo, no Estado de Mato Grosso foi identificada uma tendência decrescente da taxa de detecção de hanseníase em menores de 15 anos em um intervalo de 13 anos (2001-2013), porém crescente de casos multibacilares e com grau 2 de incapacidade física 3 .

Estudos de série temporal de hanseníase em menores de 15 anos permitem a análise da ocorrência deste fenômeno, identificando a evolução da endemia e os riscos a que as pessoas estão sujeitas, fornecendo elementos para explicações causais, auxiliando no planejamento de saúde e avaliando o impacto das ações de controle da doença já realizadas no local de estudo 7 . Permite, portanto, conhecer a magnitude, força da endemia e impacto das ações de vigilância epidemiológica da doença.

Nesse sentido, o presente traabalho teve o intuito de analisar a tendência histórica das taxas de detecção de hanseníase em menores de 15 anos no Brasil, no período de 2001 a 2016.

\section{Método}

Trata-se de um estudo ecológico com análise da tendência histórica. A série temporal é um recurso para organizar as informações quantitativas no tempo. Esse tipo de estudo deriva conhecimento sobre a distribuição das medidas de interesse em saúde, que servem para indicar os riscos a que as pessoas estão sujeitas, monitorizar a saúde da população, prever a ocorrência de eventos, for- 
necer subsídios para explicações causais, auxiliar o planejamento de saúde e avaliar o impacto das intervenções 7,8 .

A população foi constituída por menores de 15 anos, registrados no Sistema de Informação de Agravos de Notificação (SINAN) como caso novo de hanseníase. O período de estudo foi de 2001 a 2016. Para analisar séries temporais por modelos de regressão recomenda-se não utilizar menos de sete observações, dado que os testes de hipóteses apresentam baixo poder estatístico para detectar tendências nestes casos 8. Por isso, as capitais Curitiba, Florianópolis e Porto Alegre foram excluídas do estudo, devido não atingirem sete pontos para análise de tendência, por ausência de informações.

Os dados foram coletados no dia 20 de janeiro de 2017 na Sala de Apoio à Gestão Estratégica (SAGE) do Ministério da Saúde, que reúne as informações do SINAN. A SAGE é um site (http://sage. saude.gov.br) que viabiliza os indicadores de saúde, principalmente das redes de atenção à saúde prioritária, possibilitando projeções e inferências setoriais, subsidiando os dados para esta pesquisa. Esses dados passam por um processo de validação pelos diferentes entes, podendo, em alguns casos, ultrapassar um ano para consolidação/disponibilização, por isto as informações disponibilizadas referentes aos últimos anos devem ser analisadas como preliminares.

A variável de interesse foi o indicador que representa a força de morbidade e de magnitude da hanseníase (taxa de detecção de hanseníase em menores de 15 anos). A taxa detecção refere-se ao número de casos novos de hanseníase confirmados em menores de 15 anos, residentes em um determinado local e diagnosticado no ano de avaliação, dividido pela população menor de 15 anos no mesmo local e período, sendo este quociente multiplicado por 100 mil. Para a sua classificação considera-se: hiperendêmico ( $\geq 10,00$ por 100 mil habitantes); muito alto (5,00 a 9,99 por 100 mil habitantes); alto (2,50 a 4,99 por 100 mil habitantes); médio ( 0,50 a 2,49 por 100 mil habitantes); e baixo (< 0,50 por 100 mil habitantes) 9 .

As demais variáveis foram as relativas ao lugar: Brasil e suas regiões, Unidades da Federação e capitais; e as relativas ao tempo: 2001 a 2016.

Para a análise considera-se a série temporal $Y_{t}$, em que os tempos t pertencem ao conjunto $\left\{\mathrm{t}_{1}, \mathrm{t}_{2}, \ldots, \mathrm{t}_{\mathrm{n}}\right\}$. A reta de melhor ajuste para se estimar a tendência temporal é definida pela equação de regressão linear dada por $\mathrm{Y}_{\mathrm{t}}=\mathrm{b}_{0}+\mathrm{b}_{1 \mathrm{t}}+\mathrm{e}_{\mathrm{t}}$. Nessa expressão, o parâmetro $\mathrm{b}_{0}$ corresponde a uma constante, $b_{1}$ corresponde à inclinação da reta e $e_{t}$ é um erro aleatório 8 .

Para mensurar a taxa de variação da reta que ajusta os pontos da série temporal foi realizada a transformação logarítmica de base 10 dos coeficientes $(Y)$, pois contribui para a redução da heterogeneidade da variância dos resíduos da análise de regressão linear. Além disso, essa transformação contribui para a apuração da tendência. Utilizou-se o método de Prais-Winsten para a análise de regressão linear, em que os erros aleatórios $e_{t}$ incluem uma estrutura de autocorrelação temporal de primeira ordem. Nesse caso, assume-se que os erros aleatórios são dados por $\mathrm{e}=\rho \mathrm{e}_{\mathrm{t}-1}+\mathrm{w}_{\mathrm{t}}$, sendo $\mathrm{w}_{\mathrm{t}}$ um ruído branco e $|\rho|<18$.

Por meio da regressão linear foi possível estimar o valor do coeficiente $b_{1}$, aplicando-se o intervalo de confiança deste coeficiente também para o cálculo da tendência ou mudança percentual e o intervalo de confiança da medida, respectivamente. Calculou-se a estimação quantitativa da tendência pela seguinte expressão: APC $=[-1+10 \mathrm{~b} 1] * 100 \%$; e por IC95\% = $[-1+10 \mathrm{~b} 1$ mín. $] * 100 \%$; [-1 + 10b1 máx. $]$ * $100 \%$. Sendo que APC refere-se ao termo em inglês annual percent change (tendência ou mudança percentual anual) e IC ao intervalo de confiança. Quando a taxa foi positiva, a série temporal foi considerada crescente; quando negativa, foi considerada decrescente; e estacionária quando não houve diferença significante entre o seu valor e o zero ${ }^{8}$. Realizou-se o tratamento dos dados por meio do software Excel 2010 (Microsoft Corp., Estados Unidos) e a análise pelo software Stata 11.1 (StataCorp LP, College Station, Estados Unidos).

Por ser um banco de domínio público, não foi necessário submeter o projeto a um Comitê de Ética em Pesquisa. Entretanto, todas as prerrogativas éticas da Resolução no 466/2012 do Conselho Nacional de Saúde foram seguidas. 


\section{Resultados}

No período entre 2001 a 2016, a média da taxa de detecção de hanseníase em menores de 15 anos no Brasil foi de 5,77 por 100 mil habitantes, considerada muito alta. Entretanto, observa-se na Figura 1 que houve uma tendência decrescente dessa taxa, com APC de -5\% (IC95\%: -6,7; -3,3), sendo que, em 2016, a taxa de detecção foi de 2,71 por 100 mil habitantes, classificada como alta.

$\mathrm{Na}$ Figura 2, é possível constatar a série temporal da taxa de detecção de hanseníase em menores de 15 anos por região, com tendência decrescente em todas elas. A APC mais acentuada foi a da Região Sul com-10,2\% (IC95\%: -13,5; -6,8).

Entre os anos de estudo, a Região Norte manteve média hiperendêmica da taxa de detecção, com 15,63 casos por 100 mil habitantes, e o Centro-oeste e o Nordeste mantiveram média considerada muito alta, com 9,55 e 8,64 casos por 100 mil habitantes, respectivamente. Porém, a Região Sudeste apresentou média de 1,95 casos por 100 mil habitantes (média) e a Sul 0,45 casos por 100 mil habitantes (baixa).

Contudo, observa-se que, em 2016, devido à tendência decrescente, a Região Norte apresentou 7,01 casos por 100 mil habitantes (muito alto), a Centro-oeste 4,7 (alto) e a Nordeste 4,3 (alto). As regiões Sudeste e Sul mantiveram taxa de detecção média $(0,65$ por 100 mil habitantes) e baixa $(0,1$ por 100 mil habitantes), respectivamente.

A série temporal em 19 Unidades da Federação (UF) do Brasil foi decrescente, como pode ser visualizada na Tabela 1. Entretanto, entre elas, muitas se mantiveram hiperendêmicas considerando a média entre os anos estudados, como: Mato Grosso, Pará, Maranhão, Rondônia, Roraima, Pernambuco, Piauí e Acre. As UF classificadas como muito alta ou alta foram: Espírito Santo, Goiás, Amazonas, Sergipe, Ceará, Paraíba e Rio de Janeiro.

Entre as oito UF que apresentaram tendência estacionária da taxa de detecção de hanseníase em menores de 15 anos, Tocantins manteve média hiperendêmica no período, Amapá e Bahia apresentaram taxa muito alta e Alagoas alta.

As UF avaliadas como hiperendêmica no ano de 2016 foram: Tocantins (19,06 por 100 mil habitantes), Mato Grosso (13,56 por 100 mil habitantes) e Maranhão (11,42 por 100 mil habitantes). Como muito alta foram: Pará (8,74 por 100 mil habitantes), Piauí (5,74 por 100 mil habitantes) e Pernambuco (5,45 por 100 mil habitantes); e alta as UF: Rondônia (3,74 por 100 mil habitantes), Acre (3,32 por 100 mil habitantes), Amazonas (2,76 por 100 mil habitantes), Roraima (4,79 por 100 mil habitantes), Ceará (3,25 por 100 mil habitantes), Sergipe (2,65 por 100 mil habitantes), Bahia (2,59 por 100 mil habitantes) e Goiás (2,89 por 100 mil habitantes). As demais UF apresentaram taxa de detecção média (0,50 a 2,49 por 100 mil habitantes) e baixa (<0,50 por 100 mil habitantes).

Na Tabela 2, observam-se as 24 capitais brasileiras incluídas nos estudos com a tendência e a APC da taxa de detecção de hanseníase em menores de 15 anos, na qual 14 delas foram decrescentes e 10 estacionárias.

Embora com tendência decrescente dos casos novos, algumas capitais mantiveram a média da taxa de detecção hiperendêmica durante os 16 anos de estudo, como: Teresina, Recife, Cuiabá, Boa Vista, Rio Branco e Belém. Outras, porém, expuseram taxas muito altas e altas: Fortaleza, Aracaju, Vitória, Goiânia, Manaus, João Pessoa e Rio de Janeiro.

Apesar de apresentarem tendência estacionária, as capitais de Palmas e São Luís registraram a média da taxa de detecção entre os anos de 2001 e 2016 hiperendêmica. A capital Macapá, contudo, mostrou média muito alta e Salvador mostrou média alta.

Em 2016, as capitais classificadas como hiperendêmicas quanto a casos novos de hanseníase foram: Palmas (61,95 por 100 mil habitantes), Teresina (11,58 por 100 mil habitantes) e Recife (10,63 por 100 mil habitantes); classificadas como muito alta foram: Cuiabá (9,01 por 100 mil habitantes) e Fortaleza (5,81 por 100 mil habitantes); e como alta: Rio Branco (4,62 por 100 mil habitantes), Aracaju (4,93 por 100 mil habitantes), Boa Vista (3,12 por 100 mil habitantes), Porto Velho (2,99 por $100 \mathrm{mil}$ habitantes) e Manaus (2,59 por 100 mil habitantes). As demais capitais apresentaram taxa de detecção média ( 0,50 a 2,49 por 100 mil habitantes) e baixa ( $<0,50$ por 100 mil habitantes). 


\section{Figura 1}

Série temporal da taxa de detecção de hanseníase em menores de 15 anos. Brasil, 2001-2016.

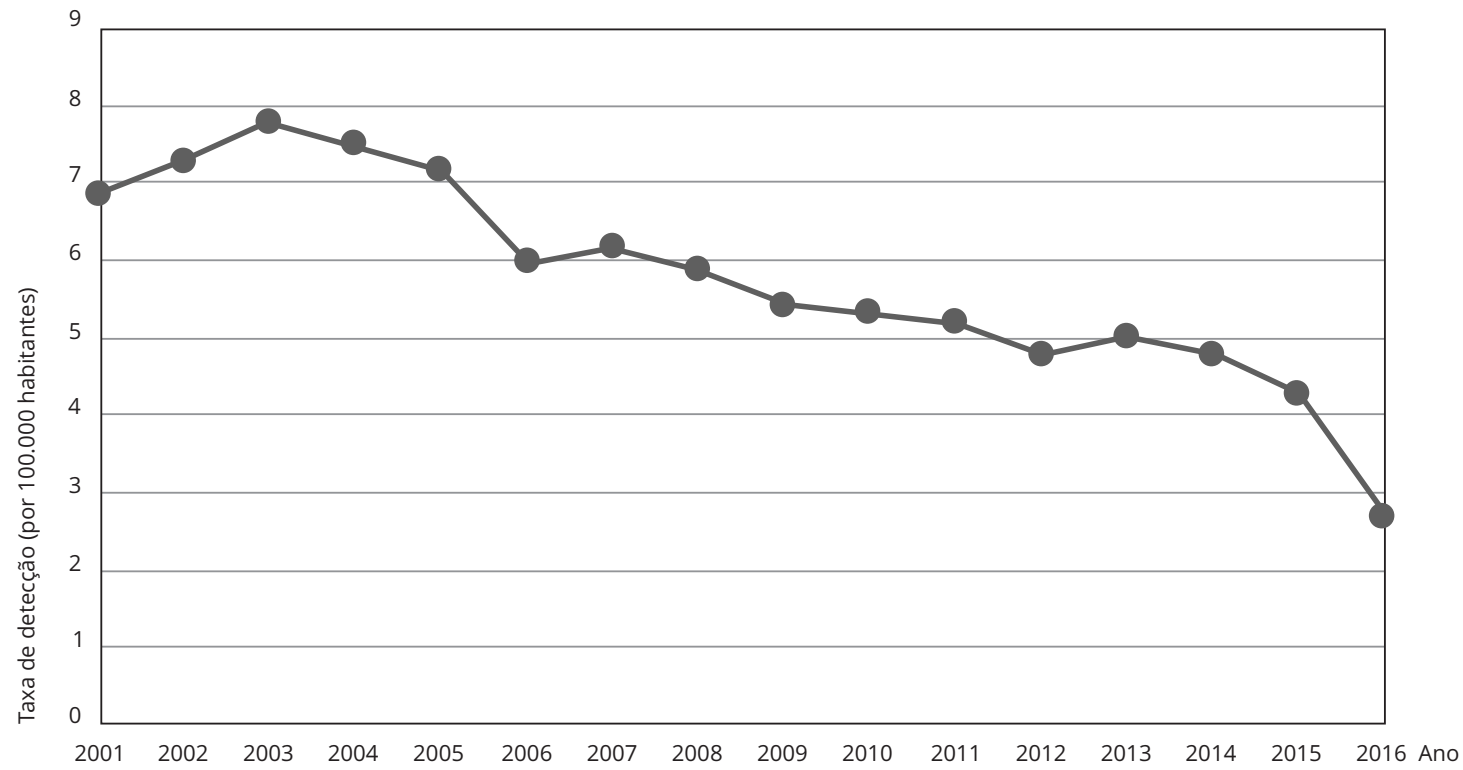

\section{Figura 2}

Série temporal da taxa de detecção de hanseníase em menores de 15 anos, por região. Brasil, 2001-2016.

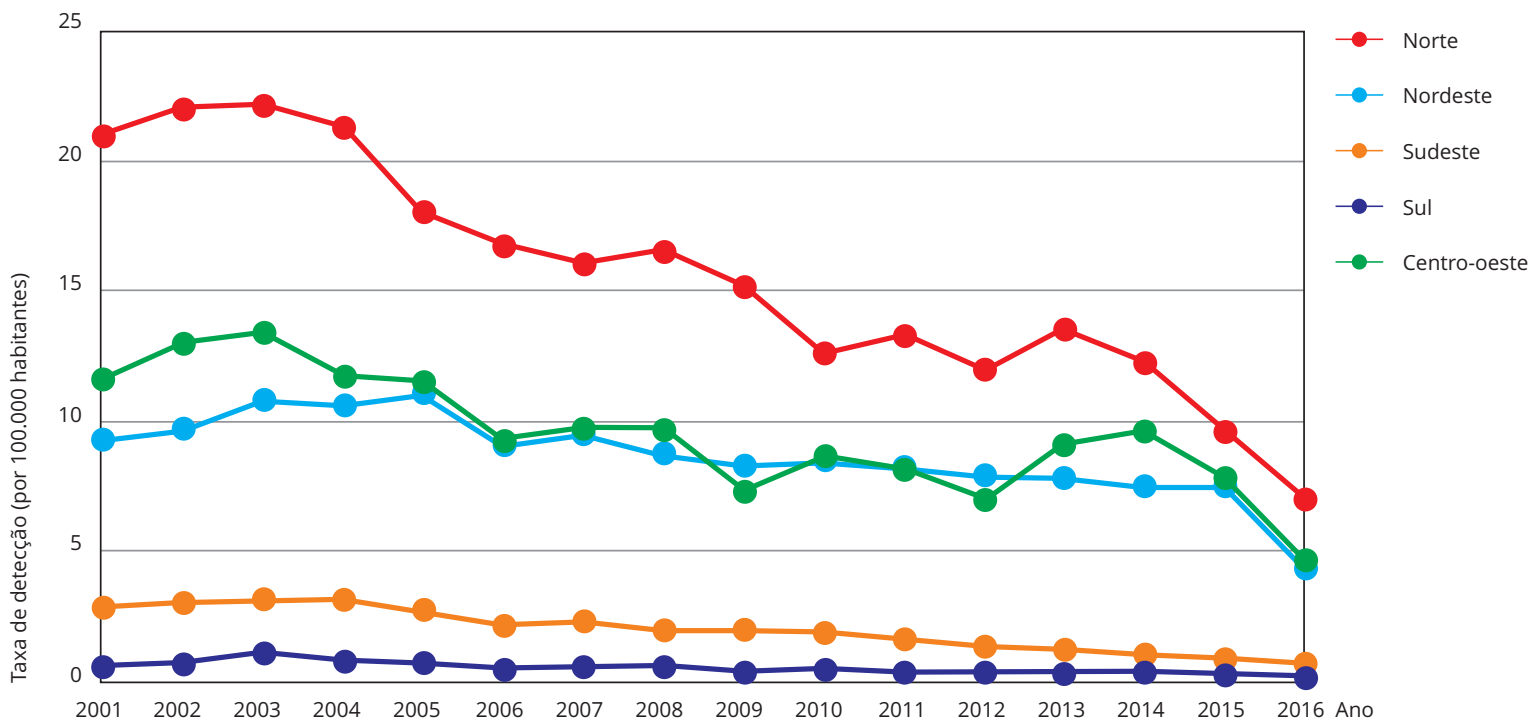


Tabela 1

Tendência e annual percent change (APC) da taxa de detecção de hanseníase em menores de 15 anos (por 100 mil habitantes), segundo a Unidade da Federação (UF). Brasil, 2001-2016.

\begin{tabular}{|c|c|c|c|c|}
\hline UF & Taxa de detecção média & APC (\%) & IC95\% & Tendência \\
\hline Rondônia & 14,12 & $-7,6$ & $-11,8 ;-3,2$ & Decrescente \\
\hline Acre & 10,86 & $-10,8$ & $-14,8 ;-6,6$ & Decrescente \\
\hline Amazonas & 7,11 & $-7,7$ & $-10,8 ;-4,5$ & Decrescente \\
\hline Roraima & 13,42 & $-16,5$ & $-20,8 ;-12,0$ & Decrescente \\
\hline Pará & 20,33 & $-6,2$ & $-8,2 ;-4,2$ & Decrescente \\
\hline Amapá & 6,10 & $-5,3$ & $-10,5 ; 0,2$ & Estacionária \\
\hline Tocantins & 23,13 & $-1,7$ & $-3,5 ; 0,2$ & Estacionária \\
\hline Maranhão & 20,17 & $-3,9$ & $-5,3 ;-2,4$ & Decrescente \\
\hline Piauí & 11,73 & $-5,2$ & $-6,7 ;-3,7$ & Decrescente \\
\hline Ceará & 5,63 & $-2,6$ & $-4,9 ;-0,2$ & Decrescente \\
\hline Rio Grande do Norte & 2,40 & $-1,6$ & $-7,1 ; 4,3$ & Estacionária \\
\hline Paraíba & 5,08 & $-9,9$ & $-15,5 ;-4,0$ & Decrescente \\
\hline Pernambuco & 12,29 & $-3,9$ & $-6,4 ;-1,3$ & Decrescente \\
\hline Alagoas & 2,60 & $-2,5$ & $-5,8 ; 1,0$ & Estacionária \\
\hline Sergipe & 5,68 & $-4,2$ & $-8,2 ;-0,1$ & Decrescente \\
\hline Bahia & 5,53 & $-2,6$ & $-5,2 ; 0,1$ & Estacionária \\
\hline Minas Gerais & 2,09 & $-9,4$ & $-11,6 ;-7,2$ & Decrescente \\
\hline Espírito Santo & 9,23 & $-9,2$ & $-14,0 ;-4,2$ & Decrescente \\
\hline Rio de Janeiro & 3,41 & $-9,9$ & $-11,9 ;-7,9$ & Decrescente \\
\hline São Paulo & 0,67 & $-9,0$ & $-13,7 ;-3,9$ & Decrescente \\
\hline Paraná & 0,86 & $-13,0$ & $-17,3 ;-8,5$ & Decrescente \\
\hline Santa Catarina & 0,29 & $-4,5$ & $-9,5 ; 0,8$ & Estacionária \\
\hline Rio Grande do Sul & 0,12 & $-5,7$ & $-9,8 ;-1,5$ & Decrescente \\
\hline Mato Grosso do Sul & 4,35 & 0,8 & $-4,6 ; 6,5$ & Estacionária \\
\hline Mato Grosso & 22,95 & $-4,0$ & $-6,3 ;-1,7$ & Decrescente \\
\hline Goiás & 7,89 & $-6,4$ & $-7,9 ;-4,9$ & Decrescente \\
\hline Distrito Federal & 1,53 & $-4,0$ & $-8,6 ; 0,8$ & Estacionária \\
\hline
\end{tabular}

IC95\%: intervalo de 95\% de confiança.

\section{Discussão}

No Brasil, embora se verifique uma redução significativa do número de casos novos em menores de 15 anos ao longo dos 16 anos de estudo, a hanseníase continua sendo uma doença de grande preocupação em saúde pública, pois apresenta, atualmente, alta endemicidade e distribuição heterogênea, com hiperendemicidade em algumas UF e capitais 2,10.

Assim como no presente trabalho, outros autores também identificaram uma tendência decrescente do número de casos novos de hanseníase em diversos países como Índia, México, Japão, Coreia do Sul e Arábia Saudita 5,11,12,13,14. Esse declínio ocorreu, possivelmente, devido ao acesso aos serviços de saúde, à oferta da poliquimioterapia (PQT) aos casos novos detectados, à busca de contatos e à imunoprofilaxia 11.

O tratamento imediato à detecção e a eficácia da PQT reduzem o número de pessoas infectadas 11. Em contrapartida, a busca ativa de contatos visa a identificar a fonte de contágio, detectar casos novos e implementar medidas preventivas, como a profilaxia pós-exposição (PPE) 15. Ambas as estratégias têm a finalidade de contribuir para a redução da cadeia de transmissão da doença.

A PPE à hanseníase é um termo amplo que engloba a imuno e quimioprofilaxia, ferramentas potencialmente poderosas e inovadoras 16. A imunoprofilaxia com a vacina Bacillus Calmette-Guérin (BCG) 
Tabela 2

Tendência e annual percent change (APC) da taxa de detecção de hanseníase em menores de 15 anos (por 100 mil habitantes), segundo a capital. Brasil, 2001-2016.

\begin{tabular}{|c|c|c|c|c|}
\hline Capital & Taxa de detecção média & APC (\%) & IC95\% & Tendência \\
\hline Porto Velho & 7,22 & $-6,8$ & $-10,5 ;-2,9$ & Decrescente \\
\hline Rio Branco & 11,15 & $-9,2$ & $-12,9 ;-5,3$ & Decrescente \\
\hline Manaus & 6,55 & $-8,0$ & $-10,5 ;-5,4$ & Decrescente \\
\hline Boa Vista & 14,18 & $-17,6$ & $-20,7 ;-14,4$ & Decrescente \\
\hline Belém & 10,17 & $-9,9$ & $-15,4 ;-4,1$ & Decrescente \\
\hline Macapá & 6,93 & $-6,5$ & $-13,0 ; 0,4$ & Estacionária \\
\hline Palmas & 25,75 & $-1,0$ & $-8,8 ; 7,5$ & Estacionária \\
\hline São Luís & 23,83 & $-1,4$ & $-4,7 ; 2,0$ & Estacionária \\
\hline Teresina & 25,47 & $-6,7$ & $-8,9 ;-4,5$ & Decrescente \\
\hline Fortaleza & 8,47 & $-2,6$ & $-4,8 ;-0,4$ & Decrescente \\
\hline Natal & 1,44 & $-0,3$ & $-7,2 ; 7,0$ & Estacionária \\
\hline João Pessoa & 6,14 & $-9,5$ & $-13,7 ;-5,2$ & Decrescente \\
\hline Recife & 26,47 & $-6,0$ & $-10,9 ;-0,9$ & Decrescente \\
\hline Maceió & 3,07 & $-3,8$ & $-9,4 ; 2,1$ & Estacionária \\
\hline Aracaju & 8,36 & $-8,6$ & $-8,6 ;-12,7$ & Decrescente \\
\hline Salvador & 4,63 & $-2,6$ & $-6,6 ; 1,6$ & Estacionária \\
\hline Belo Horizonte & 0,60 & $-3,7$ & $-9,9 ; 3,0$ & Estacionária \\
\hline Vitória & 7,80 & $-7,2$ & $-11,2 ;-3,0$ & Decrescente \\
\hline Rio de Janeiro & 2,58 & $-11,6$ & $-15,7 ;-7,4$ & Decrescente \\
\hline São Paulo & 0,54 & $-10,2$ & $-20,4 ; 1,3$ & Estacionária \\
\hline Campo Grande & 1,58 & 2,1 & $-6,4 ; 11,3$ & Estacionária \\
\hline Cuiabá & 21,69 & $-8,6$ & $-12,3 ;-4,7$ & Decrescente \\
\hline Goiânia & 6,77 & $-15,9$ & $-20,0 ;-11,5$ & Decrescente \\
\hline Brasília & 1,53 & $-4,0$ & $-8,6 ; 0,8$ & Estacionária \\
\hline
\end{tabular}

Nota: as capitais Curitiba, Florianópolis e Porto Alegre não atingiram sete pontos para análise de tendência.

é recomendada pelo Ministério da Saúde a todos os contatos, desde que não apresentem sinais e sintomas para hanseníase, sendo uma dose em caso de ausência ou presença de apenas uma cicatriz de BCG 9.

Porém, um estudo realizado no Mato Grosso, estado que apresentou média de hiperendemicidade nos anos estudados, identificou ausência de cicatriz de BCG em 4,76\% e apenas uma cicatriz de BCG em $87,14 \%$ dos contatos domiciliares e de vizinhança, demonstrando que a imunoprofilaxia não está sendo implementada no estado conforme preconizado pelo Ministério da Saúde 17. A ausência de cicatriz de BCG implica um risco 3,7 vezes maior de ocorrência de hanseníase do que aqueles cuja cicatriz está presente 18. Esse episódio é uma das barreiras para a eliminação da doença e manutenção da endemicidade.

Por sua vez, a quimioprofilaxia é feita por meio de um dos medicamentos que compõe a PQT, sendo a rifampicina dose única a medicação com o melhor efeito comprovado 19. Em 2015, a Portaria no 32/2015, do Ministério da Saúde, tornou pública a decisão de incorporar a quimioprofilaxia de contatos de doentes de hanseníase com rifampicina dose única no âmbito do Sistema Único de Saúde (SUS). Todavia, essa intervenção está sendo realizada e analisada, a priori, apenas em alguns municípios dos estados de Pernambuco, Mato Grosso e Tocantins, que configuram importantes áreas endêmicas para a doença no Brasil 20. A expansão dessa estratégia as outras localidades pode possibilitar um declínio dos casos de hanseníase, tendo em vista o seu potencial de prevenção do desenvolvimento da doença.

As melhorias socioeconômicas e ecológicas também são fatores que contribuíram para a redução da carga da doença, uma vez que as condições socioeconômicas precárias influenciam no nível de higiene, no aglomeramento dentro das famílias, na baixa escolaridade, nas condições gerais de 
saúde, desnutrição e movimentos migratórios, que são fatores associados à hanseníase 14,21. Pois, nos últimos anos, o Brasil apresentou mudanças sociais importantes caracterizadas, especialmente, pela diminuição da desigualdade e da pobreza 22 . Todavia, ainda há disparidades a serem enfrentadas, que resultariam em mais avanços no controle da doença.

Um dos fatores apontados, especificamente para a queda da taxa de detecção em menores de 15 anos, é a mudança na distribuição etária dos casos detectados, com o deslocamento para os grupos etários mais avançados 14 . A taxa de detecção de hanseníase nessa faixa etária é considerada o principal indicador da endemia, necessária para se conhecer a magnitude e o desempenho do sistema de saúde para a eliminação da doença. Espera-se que a taxa de detecção de hanseníase em menores de 15 anos seja abaixo de 0,50 casos por 100 mil habitantes, pois seus índices altos são preocupantes, uma vez que a doença é infectocontagiosa e tem poder incapacitante e estigmatizante 3,9.

Como mencionado anteriormente, o país apresenta hiperendemicidade em uma quantidade considerável de UF e capitais, o que indica a persistência do bacilo na comunidade, fonte de transmissibilidade contínua e precoce, e dificuldades no controle e vigilância da doença nessas localidades 3 .

A distribuição heterogênea da doença pode ser uma resposta aos fatores de capacidade diagnóstica, à vulnerabilidade territorial pela presença de fontes de transmissibilidade e às desigualdades socioeconômicas no acesso aos serviços de saúde no país 21,22. Um estudo apontou que, entre os anos de 1990 e 2009, o coeficiente de Gini, que mede a desigualdade de renda e a taxa de pobreza, reduziu significativamente no país, sendo que as regiões Sul e Sudeste apresentaram as maiores quedas, o que pode explicar o declínio mais acentuado da hanseníase nestas regiões e as menores taxas de detecção identificadas 22 .

Assim como neste estudo, Magalhães \& Rojas 21 detectaram maior concentração de casos pela agregação de vários municípios no centro-norte do país, pertencentes aos estados da Região Norte, Centro-oeste e Nordeste. Os autores registraram a coincidência entre municípios com altas taxas de detecção e rodovias construídas após a década de 1970, todas federais, que facilitaram o processo de ocupação da fronteira agrícola norte do país e, logo, o surgimento de assentamentos, geralmente urbanos e sedes de novos municípios. Acredita-se que a expansão da fronteira agrícola promoveu a expansão espacial da endemia.

Algumas localidades apresentaram tendência estacionária com elevada taxa de detecção ao longo dos 16 anos de estudo, indicando que não houve progresso nas ações de controle da doença e, portanto, estas regiões não vêm atuando de maneira efetiva e resolutiva, sendo necessários ajustes e investimentos no planejamento em saúde para alcançar a eliminação da hanseníase.

O Brasil se mantém como o segundo país mais atingido pela hanseníase no mundo e o primeiro na América, precisando de progressos para atingir a meta de eliminação da doença, estabelecidos pela OMS 2. Para isso, o programa de eliminação da hanseníase deve estar entre as ações prioritárias do Ministério da Saúde e continuar a ser implantado em todas as localidades brasileiras, com foco nas áreas endêmicas. Esse deve propor atividades de educação em saúde, investigação epidemiológica para o diagnóstico oportuno de casos, provisão contínua de PQT para o tratamento até a cura, prevenção e tratamento de incapacidades físicas, vigilância epidemiológica, exame de contatos, orientações e a implementação de PPE, conforme mencionado nas Diretrizes para Vigilância, Atenção e Eliminação da Hanseníase como um Problema de Saúde Pública 9.

Por trabalhar com dados públicos, o trabalho apresenta limitações como a possibilidade de problemas no fluxo de recebimento de informações, de erros durante a transferência dos dados e de subnotificação. Além disso, as informações utilizadas referentes aos últimos anos são preliminares, podendo assumir valores mais baixos do que os reais. Portanto, as taxas de detecção apresentadas dos últimos anos podem ser maiores do que os valores apresentados neste estudo, pois podem ultrapassar um ano para a consolidação.

Recomenda-se a realização de novos trabalhos que avaliem a tendência da taxa de detecção de casos novos de hanseníase em menores de 15 anos por classificação operacional, tendo em vista que alguns estudos apontam que, embora se verifique tendência decrescente da taxa de detecção de casos novos, muitas vezes a tendência da detecção de casos multibacilares apresenta-se crescente 3,14. Assim como da tendência dos indicadores de proporção de contatos intradomiciliares avaliados e proporção de cura, que determinam a qualidade das ações e serviços 3 . 


\section{Conclusão}

A análise da tendência das taxas de detecção de hanseníase em menores de 15 anos no Brasil entre 2001 e 2016 foi decrescente, porém, observou-se a presença de hiperendemicidade em algumas UF e capitais brasileiras, principalmente na região centro-norte, o que demonstra permanência de fontes de transmissibilidade e dificuldade na eliminação da hanseníase. Algumas localidades apresentaram tendência estacionária com elevada taxa de detecção em 16 anos de estudo, indicando deficiência na implantação de ações de controle da doença, necessitando maior planejamento em saúde. Nesse sentido, cabe aos profissionais de saúde desenvolver o plano para a redução da carga de hanseníase em sua área de abrangência por meio da educação em saúde, investigação epidemiológica para a detecção precoce de casos novos, tratamento oportuno, prevenção e tratamento de incapacidades, e vigilância epidemiológica.

\section{Colaboradores}

P. B. Schneider contribuiu com a coleta, análise e interpretação dos dados, redação e aprovação da versão final do manuscrito. B. H. B. M. Freitas contribuiu com a concepção e delineamento do estudo, interpretação dos dados, redação e aprovação da versão final do manuscrito. Ambas as autoras são responsáveis por todos os aspectos do trabalho, incluindo a garantia de sua precisão e integridade.

\section{Referências}

1. Secretaria de Vigilância em Saúde, Ministério da Saúde. Hanseníase, verminoses e tracoma têm cura: a experiência de uma campanha integrada. Boletim Epidemiológico 2016; 47(21):1-10

2. World Health Organization. Global leprosy update, 2015: time for action, accountability and inclusion. Wkly Epidemiol Rec 2016; 91:405-20.

3. Freitas BHBM, Cortela DCB, Ferreira SMB. Tendência da hanseníase em menores de 15 anos em Mato Grosso (Brasil), 2001-2013. Rev Saúde Pública 2017; 51:28.

4. Departamento de Articulação Interfederativa, Secretaria de Gestão Estratégica e Participativa, Ministério da Saúde. Caderno de diretrizes, objetivos, metas e indicadores: 2013-2015. 2a Ed. Brasília: Ministério da Saúde; 2014.

5. Sasidharanpillai S, Binitha MP, Riyaz N, Ambooken B, Mariyath OK, George B, et al. Children leprosy: a retrospective descriptive study from Government Medical College, Kozhikode, Kerala, India. Lepr Rev 2014; 85:100-10.

6. Alencar CHM, Barbosa JC, Ramos Jr. AN, Alencar MJF, Pontes RJS, Castro CGJ, et al. Hanseníase no Município de Fortaleza - CE, Brasil: aspectos epidemiológicos e operacionais em menores de 15 anos (1995-2007). Rev Bras Enferm 2008; 61:694-700.

7. Pereira MG. Variáveis relativas ao tempo. In: Pereira MG, organizador. Epidemiologia: teoria e prática. Rio de Janeiro: Guanabara Koogan; 1995; p. 245-67.

8. Antunes JLF, Cardoso MRA. Uso da análise de séries temporais em estudos epidemiológicos. Aplicações da epidemiologia. Epidemiol Serv Saúde 2015; 24:565-76.

9. Departamento de Vigilância das Doenças Transmissíveis, Secretaria de Vigilância em Saúde, Ministério da Saúde. Diretrizes para vigilância, atenção e eliminação da hanseníase como problema de saúde pública: manual técnico-operacional. Brasília: Ministério da Saúde; 2016. 
10. Levantezi M, Moreira T, Sena Neto S, De Jesus AL. Leprosy in children under fifteen years in Brazil, 2011. Lepr Rev 2014; 85:118-22.

11. Assiri A, Yezli S, Tayeb T, Almasri M, Bamgboye AE, Memish ZA. Eradicating leprosy in Saudi Arabia: outcome of a tem-year surveillance (2003-2012). Travel Med Infect Dis 2014; $12: 771-7$

12. Koba A, Ishii N, Mori S, Fine PE. The decline of leprosy in Japan: patterns and trends 19642008. Lepr Rev 2009; 80:432-40.

13. Larrea MR, Carreno MC, Fine PE. Patterns and trends of leprosy in Mexico: 1989-2009. Lepr Rev 2012; 83:184-94.

14. Lee J, Kim JP, Nishikiori N, Fine PE. The decline of leprosy in the Republic of Korea: patterns and trends 1977-2013. Lepr Rev 2015; 86:316-27.

15. Lobato DC, Neves DCO, Xavier MB. Avaliação das ações da vigilância de contatos domiciliares de pacientes com hanseníase no Município de Igarapé-Açu, Estado do Pará, Brasil. Rev Pan-Amazônica Saúde 2016; 7:45-53.

16. Richardus RA, Alam K, Pahan D, Feenstra SG, Geluk A, Richardus JH. The combined effect of chemoprophylaxis with single dose rifampicin and immunoprophylaxis with BCG to prevent leprosy in contacts of newly diagnosed leprosy cases: a cluster randomized controlled trial (MALTALEP study). BMC Infect Dis 2013; 13:456-64.
17. Gomes LC. Infecção por Mycobacterium leprae em menores de 15 anos contatos de casos de hanseníase [Dissertação de Mestrado]. Cuiabá: Universidade Federal de Mato Grosso; 2016.

18. Goulart IMB, Souza DOB, Marques CR, Pimenta VL, Gonçalves MA, Goulart LR. Risk and protective factors for leprosy development determined by epidemiological surveillance of household contacts. Clin Vaccine Immunol 2008; 15:101-5.

19. Moet FJ, Pahan D, Oskam L, Richardus JH; COLEP Study Group. Effectiveness of single dose rifampicin in preventing leprosy in close contacts of patients with newly diagnosed leprosy: cluster randomised controlled trial. $\mathrm{Br} \mathrm{J}$ Cancer 2008; 336:761-4.

20. Comissão Nacional de Incorporação de Tecnologias do SUS. Quimioprofilaxia de contatos de doentes de hanseníase com rifampicina em dose única. Brasília: Ministério da Saúde; 2015.

21. Magalhães MCC, Rojas LI. Diferenciação territorial da hanseníase no Brasil. Epidemiol Serv Saúde 2007; 16:75-84.

22. Andrade MV, Noronha KVMS, Menezes RM, Souza MN, Reis CB, Martins DR, et al. Desigualdade socioeconômica no acesso aos serviços de saúde no Brasil: um estudo comparativo entre as regiões brasileiras em 1998 e 2008. Economia Aplicada 2013; 17:623-45. 


\section{Abstract}

The aim of this study was to analyze trends in the detection rate of leprosy in children under 15 years of age in Brazil from 2001 to 2016. A time series study used the Prais-Winsten procedure for generalized linear regression analysis, with significance set at 5\%. The mean detection rate was $5.77 \mathrm{per}$ 100,000 inhabitants. However, a downward trend was observed in this rate, with an annual percent change (APC) of -5\% (95\%CI: - 6.7; -3.3). There was a downward trend in all regions of Brazil. Among the regions, the North maintained a hyperendemic mean detection rate $(\geq 10.00$ per 100,000 inhabitants). The time series was downward in 19 states of Brazil. However, many states maintained a hyperendemic mean rate, such as Mato Grosso, Pará, Maranhão, Rondônia, Roraima, Pernambuco, Piaui, and Acre. Among the eight states that maintained a stationary trend, Tocantins had a hyperendemic mean rate during the period analyzed. Of the 24 Brazilian state capitals included in the study, 14 showed downward trends and 10 stationary trends. Despite the downward trend in new cases, some state capitals maintained hyperendemic mean rates (Teresina, Recife, Cuiabá, Boa Vista, Rio Branco, and Belém). Despite stationary trends, the state capital cities Palmas and São Luís recorded hyperendemic mean rates. In conclusion, although the analysis showed a downward trend in Brazil, there were stationary trends and hyperendemicity in some states and in some state capitals, highlighting the persistence of sources of transmissibility and difficulty in eliminating leprosy from the country.

Leprosy; Child; Adolescent; Temporal Distribution

\section{Resumen}

El objetivo fue analizar la tendencia de las tasas de detección de hanseniasis en menores de 15 años en Brasil, durante el periodo de 2001 a 2016. Se trata de un estudio de serie temporal, utilizando el procedimiento de Prais-Winsten para el análisis de regresión lineal generalizada con un nivel de significancia de un 5\%. La media de la tasa de detección fue de un 5,77 por 100 mil habitantes. No obstante, se observó una tendencia decreciente de esa tasa, con el annual percent change (APC) de -5\% (IC95\%: - 6, 7; -3,3). Se verificó la tendencia decreciente en todas las regiones del país. Entre las regiones, la Norte fue la que mantuvo la media hiperendémica $(\geq 10,00$ por 100 mil habitantes) de la tasa de detección. La serie temporal en 19 Unidades de la Federación (UF) fue decreciente. Todavia, entre ellas, muchas mantuvieron una media hiperendémica, como: Mato Grosso, Pará, Maranhão, Rondônia, Roraima, Pernambuco, Piauí y Acre. Entre las ocho UF que presentaron una tendencia estacionaria, Tocantins mantuvo una media hiperendémica en este período. De las 24 capitales brasileñas incluidas en el estudio, 14 de ellas fueron decrecientes y 10 estacionarias. Pese a la tendencia decreciente de los nuevos casos, algunas capitales mantuvieron una media hiperendémica como: Teresina, Recife, Cuiabá, Boa Vista, Rio Branco y Belém. A pesar de que presentaron una tendencia estacionaria, las capitales Palmas y São Luís registraron una media hiperendémica. Se concluyó que, aunque el análisis de la tendencia fue decreciente en Brasil, hubo presencia de tendencias estacionarias e hiperendemicidad en algunas UF y capitales brasileñas, lo que demuestra la permanencia de fuentes de transmisibilidad y dificultad en la eliminación de la hanseniasis en el país.

Lepra; Niño; Adolescente; Distribución Temporal
Recebido em 13/Jun/2017

Versão final reapresentada em 20/Set/2017

Aprovado em 02/Out/2017 